\title{
19. INTERLABORATORY COMPARISON OF LEG 46 BASALT STANDARDS
}

\author{
R. James Kirkpatrick, Scripps Institution of Oceanography, La Jolla, California ${ }^{1} 92093$ \\ and \\ The Leg 46 Shipboard Scientific' Party $^{2}$
}

Because a number of laboratories planned to analyze Leg 46 basalts, three samples from Hole 396B were selected for interlaboratory comparison. These are: (1) Sample 7-1, $50-57 \mathrm{~cm}$, a sparsely phyric pillow basalt with olivine and plagioclase phenocrysts from chemical unit A-1, (2) Sample $15-2,120-133 \mathrm{~cm}$, a piece of sparsely phyric cooling unit in chemical unit A-3, and (3) Sample 22-3, 28-38 cm, a phyric pillow basalt with olivine and plagioclase phenocrysts from chemical unit B-2. All samples appeared in hand specimen to be among the freshest available.

All three samples were returned to the NASA-Johnson Space Center in Houston, Texas, and were powdered and homogenized there. Each investigator was then sent a 25 gram split.

Six laboratories analyzed the three samples for major elements. Tables 1, 2 and 3 present the analyses as reported. The six laboratories are Centre Océanologique de Bretagne, Brest, France (COB, Bougault et al., this volume); the Department of Earth Sciences of Kanazawa University, Kanazawa, Japan (KAN, Sato, et al., this volume); the Laboratoire de Petrologie, Universite de Nancy 1, Nancy, France (UN-1, Mevel et al., this volume); the NASAJohnson Space Center, Houston, Texas (JSC, Dungan et al., this volume); the Institut für Mineralogie, RuhrUniversität Bochum, Bochum, Federal Republic of Germany (R-UB, Flower et al., this volume); and the Vernadsky Institute of Geochemistry Academy of Sciences of the USSR, Moscow, USSR (VIG, Dmitriev, this volume). The XRF laboratory aboard the Glomar Challenge did not analyze the standards, but did analyze Samples 7-1, 53-55 $\mathrm{cm}$, and 15-2, 129-131 cm, which are from the same intervals as standards 1 and 2 . These analyses are included in Tables 1 and 2, respectively.

'Present address: Dept. of Geology, Univ. of Illinois, Urbana, Illinois.

${ }^{2}$ J.R. Heirtzler, L. Dmitriev, P. Cambon, H. Dick, M. Dungan, A. Erickson, F.N. Hodges, J. Honnorez, D. Matthews, D. Ohnenstetter, N. Petersen, H. Sato, H. Schmincke.
To facilitate comparison of the analyses each was recalculated to a dry and reduced state and then normalized to 100 per cent. These values are presented in Tables 4, 5, and 6, along with means, standard deviations, and the standard deviation as per cent of the mean. The shipboard analyses are not included in these averages because they were not done on the homogenized standard powders.

In general, the analyses from different laboratories are in good agreement with each other. As observed by Wright (1977) for the Leg 37 standards, however, there are some small but consistent differences between laboratories. These differents are not nearly large enough to affect the overall classification of the rocks or in most cases even the relative amounts of elements. The differences are large enough, though, to cause some difficulties with very detailed work, such as identifying chemical stratigraphy within major lithologic and chemical units. For some elements the variation between laboratories for one sample is as large or larger than the total variation found by the shipboard laboratory in the chemical sub-unit from which the sample came. This is illustrated by the $\mathrm{FeO}^{*}-\mathrm{MgO}$ plot in Figure 1. Great care is needed, then, in using analyses from different laboratories for such detailed work, and it seems best to use those from one laboratory for this kind of work.

Two laboratories analyzed the samples for trace elements. These data are presented in Table 7. These laboratories are the NASA-Johnson Space Center, Houston, Texas (JSC, Dungan et al., this volume) and the Institut für Petrographie und Geochemie der Universität (T.H.) Karlsruhe, Federal Republic of Germany (KAR, Emmerman and Puchelt, this volume). The agreement between the two laboratories is excellent.

\section{REFERENCE}

Wright, T.L., 1977. Chemical Analyses of Interlaboratory Standards, In Aumento, F., Melson, W.G., et al., Initial Reports of the Deep Sea Drilling Project, Volume 37: Washington (U.S. Government Printing Office), p. 367-370. 
TABLE 1

As Reported Major Element Analyses of Leg 46 Basalt Standard \#1-Sample 396B-7-1, 50-57 cm

\begin{tabular}{|c|c|c|c|c|c|c|c|c|c|}
\hline \multirow[b]{2}{*}{$\mathrm{SiO}_{2}$} & \multicolumn{2}{|c|}{ СОВ } & \multirow{2}{*}{$\frac{\text { KAN }}{48.98}$} & \multirow{2}{*}{$\frac{\text { UN-I }}{48.96}$} & \multirow{2}{*}{$\begin{array}{r}\text { JSC } \\
49.24\end{array}$} & \multirow{2}{*}{$\begin{array}{l}\text { R-UB } \\
49.95\end{array}$} & \multicolumn{2}{|c|}{ VIG } & \multirow{2}{*}{$\begin{array}{c}\mathrm{GC}^{\mathrm{a}} \\
49.42\end{array}$} \\
\hline & 49.30 & 49.41 & & & & & 48.45 & 48.70 & \\
\hline $\mathrm{TiO}_{2}^{2}$ & 1.41 & 1.43 & 1.50 & 1.42 & 1.40 & 1.42 & 1.42 & 1.42 & 1.42 \\
\hline $\mathrm{Al}_{2} \mathrm{O}_{3}$ & 15.64 & 15.86 & 15.47 & 15.28 & 15.46 & 15.77 & 15.73 & 15.63 & 15.63 \\
\hline $\mathrm{Fe}_{2} \mathrm{O}_{3}$ & & & 3.11 & & 3.58 & 2.85 & 3.13 & 3.16 & \\
\hline $\mathrm{Fe}_{2} \mathrm{O}_{3} \mathrm{~b}$ & 10.43 & 10.55 & & 10.23 & & & & & 10.47 \\
\hline $\mathrm{FeO}$ & & & 6.54 & & 5.98 & 6.76 & 6.89 & 6.70 & \\
\hline $\mathrm{MgO}$ & 8.03 & 7.98 & 7.71 & 8.26 & 7.72 & 8.10 & 7.97 & 8.04 & 8.03 \\
\hline $\mathrm{CaO}$ & 11.89 & 12.00 & 11.76 & 11.76 & 11.63 & 12.03 & 11.50 & 11.45 & 11.82 \\
\hline $\mathrm{Na}_{2} \mathrm{O}$ & 2.46 & 2.39 & 2.41 & 2.61 & 2.54 & 2.41 & 2.65 & 2.69 & 2.56 \\
\hline $\mathrm{K}_{2} \mathrm{O}$ & 0.19 & 0.19 & 0.24 & 0.20 & 0.18 & 0.22 & 0.24 & 0.24 & 0.19 \\
\hline $\mathrm{MnO}$ & 0.17 & 0.17 & 0.17 & $(0.20)$ & 0.16 & 0.17 & 0.17 & 0.17 & 0.19 \\
\hline $\mathrm{P}_{2} \mathrm{O}_{5}$ & 0.14 & 0.16 & 0.15 & & 0.11 & 0.14 & & & 0.14 \\
\hline $\mathrm{S}^{2}$ & & & & & 0.06 & & & & \\
\hline $\mathrm{CO}_{2}$ & & & & & & 0.06 & & & 0.23 \\
\hline L.O.I. & & & & 0.90 & & & & & \\
\hline $\mathrm{H}_{2} \mathrm{O}^{+}$ & 0.45 & 0.45 & 1.25 & & & 0.63 & & & 0.81 \\
\hline $\mathrm{H}_{2} \mathrm{O}^{-}$ & 0.54 & 0.54 & 0.65 & & & 0.60 & & & \\
\hline $\mathrm{H}_{2} \mathrm{O}$ total & & & & & 1.64 & & 1.20 & 1.10 & \\
\hline
\end{tabular}

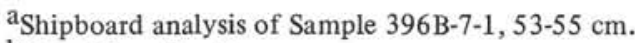

b $\mathrm{Total} \mathrm{Fe}$ as $\mathrm{Fe}_{2} \mathrm{O}_{3}$.

${ }^{c}$ Dry totals, not including any $\mathrm{H}_{2} \mathrm{O}$ or $\mathrm{CO}_{2}$.

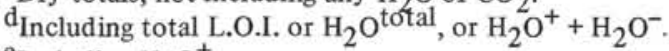

' Including $\mathrm{H}_{2} \mathrm{O}^{+}$.

TABLE 2

As Reported Major Element Analyses of Leg 46 Basalt Standard \#2-Sample 396B-15-2, 120-133 cm

\begin{tabular}{|c|c|c|c|c|c|c|c|c|c|}
\hline \multirow[b]{2}{*}{$\mathrm{SiO}_{2}$} & \multicolumn{2}{|c|}{$\mathrm{COB}$} & \multirow{2}{*}{$\begin{array}{l}\text { KAN } \\
48.86\end{array}$} & \multirow{2}{*}{$\begin{array}{r}\text { UN-I } \\
48.61\end{array}$} & \multirow{2}{*}{$\begin{array}{r}\text { JSC } \\
49.02\end{array}$} & \multirow{2}{*}{$\begin{array}{c}\text { R-UB } \\
49.69\end{array}$} & \multicolumn{2}{|c|}{ VIG } & \multirow{2}{*}{$\frac{\mathrm{GC}^{\mathrm{a}}}{49.90}$} \\
\hline & 49.31 & 49.37 & & & & & 48.64 & 48.34 & \\
\hline $\mathrm{TiO}_{2}$ & 1.64 & 1.64 & 1.75 & 1.61 & 1.61 & 1.62 & 1.60 & 1.64 & 1.63 \\
\hline $\mathrm{Al}_{2} \mathrm{O}_{3}$ & 15.28 & 15.28 & 15.11 & 14.93 & 14.97 & 15.26 & 15.29 & 15.29 & 14.98 \\
\hline $\mathrm{Fe}_{2} \mathrm{O}_{3}$ & & & 2.75 & & 2.20 & 3.32 & 3.38 & 3.08 & \\
\hline $\mathrm{Fe}_{2} \mathrm{O}_{3} *$ & 11.30 & 11.21 & & 10.88 & & & & & 11.20 \\
\hline $\mathrm{FeO}$ & & & 7.26 & & 7.68 & 6.92 & 7.24 & 7.35 & \\
\hline $\mathrm{MgO}$ & 8.11 & 8.19 & 7.88 & 8.35 & 7.96 & 8.02 & 7.78 & 7.99 & 7.93 \\
\hline $\mathrm{CaO}$ & 10.99 & 10.99 & 10.49 & 10.69 & 10.58 & 10.95 & 10.52 & 10.45 & 11.02 \\
\hline $\mathrm{Na}_{2} \mathrm{O}$ & 2.63 & 2.56 & 2.82 & 2.89 & 2.97 & 2.69 & 3.02 & 3.03 & 2.68 \\
\hline $\mathrm{K}_{2} \mathrm{O}$ & 0.13 & 0.13 & 0.18 & 0.13 & 0.12 & 0.14 & 0.18 & 0.18 & 0.14 \\
\hline $\mathrm{MnO}$ & 0.16 & 0.16 & 0.16 & $(0.19)$ & 0.15 & 0.16 & 0.15 & 0.15 & 0.17 \\
\hline $\mathrm{P}_{2} \mathrm{O}_{5}$ & 0.14 & 0.15 & 0.18 & & 0.13 & 0.15 & & & 0.17 \\
\hline $\mathrm{S}$ & & & & & 0.12 & & & & \\
\hline $\mathrm{CO}_{2}$ & & & & & & 0.02 & & & 0.13 \\
\hline L.O.I. & & & & 1.59 & & & & & \\
\hline $\mathrm{H}_{2} \mathrm{O}^{+}$ & 0.80 & 0.80 & 1.49 & & & 0.79 & & & 0.90 \\
\hline $\mathrm{H}_{2}^{-} \mathrm{O}^{-}$ & 0.91 & 0.91 & 1.18 & & & & & & \\
\hline $\mathrm{H}_{2} \mathrm{O}^{\text {total }}$ & & & & & 2.35 & & 1.88 & 1.73 & \\
\hline Total & $99.69^{b}$ & $99.68^{b}$ & $100.11^{c}$ & $99.87^{c}$ & $99.87^{\mathrm{c}}$ & $99.73^{\mathrm{d}}$ & $99.68^{\mathrm{c}}$ & $99.23^{\mathrm{c}}$ & $99.81^{b}$ \\
\hline
\end{tabular}

${ }^{\text {a }}$ Shipboard analy sis of Sample 396B-15-2, 129-131 cm.

bry totals, not including any $\mathrm{H}_{2} \mathrm{O}$ or $\mathrm{CO}_{2}$.

Including total L.O.I. or $\mathrm{H}_{2} \mathrm{O}$ total or $\mathrm{H}_{2} \mathrm{O}^{+}+\mathrm{H}_{2} \mathrm{O}^{-}$.

${ }^{\mathrm{d}}$ Including $\mathrm{H}_{2} \mathrm{O}^{+}$. 
TABLE 3

As Reported Major Element Analyses of Leg 46 Basalt Standard \#3-Sample 396B-22-3, $28-38 \mathrm{~cm}$

\begin{tabular}{|c|c|c|c|c|c|c|c|c|}
\hline \multirow[b]{2}{*}{$\mathrm{SiO}_{2}$} & \multicolumn{2}{|c|}{$\mathrm{COB}$} & \multirow{2}{*}{$\frac{\text { KAN }}{48.27}$} & \multirow{2}{*}{$\begin{array}{c}\text { UN-I } \\
48.58\end{array}$} & \multirow{2}{*}{$\frac{\text { JSC }}{48.24}$} & \multirow{2}{*}{$\frac{\text { R-UB }}{49.06}$} & \multicolumn{2}{|c|}{ VIG } \\
\hline & 48.62 & 48.79 & & & & & 48.32 & 48.09 \\
\hline $\mathrm{TiO}_{2}$ & 0.98 & 0.97 & 1.65 & 0.96 & 0.96 & 0.96 & 1.00 & 1.00 \\
\hline $\mathrm{Al}_{2} \mathrm{O}_{3}$ & 18.11 & 18.05 & 17.30 & 17.16 & 17.55 & 17.79 & 17.72 & 17.82 \\
\hline $\mathrm{Fe}_{2} \mathrm{O}_{3}$ & & & 3.20 & & 2.96 & 3.18 & 2.83 & 2.94 \\
\hline $\mathrm{Fe}_{2} \mathrm{O}_{3} *$ & 8.51 & 8.51 & & 8.01 & & & & \\
\hline $\mathrm{FeO}$ & & & 4.08 & & 4.81 & 4.73 & 5.25 & 5.15 \\
\hline $\mathrm{MgO}$ & 8.15 & 8.02 & 7.81 & 8.39 & 7.75 & 8.04 & 8.00 & 8.15 \\
\hline $\mathrm{CaO}$ & 12.54 & 12.46 & 11.99 & 12.03 & 12.12 & 12.34 & 11.58 & 11.72 \\
\hline $\mathrm{Na}_{2} \mathrm{O}$ & 2.12 & 2.22 & 2.35 & 2.51 & 2.43 & 2.28 & 2.52 & 2.53 \\
\hline $\mathrm{K}_{2} \mathrm{O}$ & 0.18 & 0.18 & 0.23 & 0.19 & 0.18 & 0.17 & 0.23 & 0.24 \\
\hline $\mathrm{MnO}$ & 0.13 & 0.13 & 0.14 & $(0.15)$ & 0.13 & 0.13 & 0.14 & 0.14 \\
\hline $\mathrm{P}_{2} \mathrm{O}_{5}$ & 0.09 & 0.09 & 0.14 & & 0.07 & 0.09 & & \\
\hline $\mathrm{s}^{2}$ & & & & & 0.02 & & & \\
\hline $\mathrm{CO}_{2}$ & & & & & & 0.03 & & \\
\hline L.O.I. & & & & 1.81 & & & & \\
\hline $\mathrm{H}_{2} \mathrm{O}^{+}$ & 0.90 & 0.90 & 1.58 & & & 0.80 & & \\
\hline $\mathrm{H}_{2}^{-} \mathrm{O}^{-}$ & 0.96 & 0.96 & 1.09 & & & & & \\
\hline $\mathrm{H}_{2}^{2} \mathrm{O}^{\text {total }}$ & & & & & 2.19 & & 1.81 & 1.78 \\
\hline Total & $99.43^{\mathrm{a}}$ & $99.42^{\mathrm{a}}$ & $99.83^{b}$ & $99.79^{b}$ & $99.41^{b}$ & $99.60^{c}$ & $99.40^{\mathrm{b}}$ & $99.56^{\mathrm{b}}$ \\
\hline
\end{tabular}

a Dry totals, not including any $\mathrm{H}_{2} \mathrm{O}$ or $\mathrm{CO}_{2}$.

${ }^{b}$ Including total L.O.I. or $\mathrm{H}_{2} \mathrm{O}^{\text {total }}$, or $\mathrm{H}_{2} \mathrm{O}^{+}+\mathrm{H}_{2} \mathrm{O}^{-}$.

${ }^{\mathrm{c}}$ Including $\mathrm{H}_{2} \mathrm{O}^{+}$

TABLE 4

Dry-Reduced Analyses of Leg 46 Basalt Standard \#1-Sample 396B-7-1, 50-57 cm

\begin{tabular}{|c|c|c|c|c|c|c|c|c|c|c|c|c|}
\hline \multirow[b]{2}{*}{$\mathrm{SiO}_{2}$} & \multicolumn{2}{|c|}{$\mathrm{COB}$} & \multirow{2}{*}{$\frac{\mathrm{KAN}}{50.11}$} & \multirow{2}{*}{$\frac{\text { UN-I }}{50.01}$} & \multirow{2}{*}{$\begin{array}{l}\text { JSC } \\
50.40\end{array}$} & \multirow{2}{*}{$\begin{array}{l}\text { R-UB } \\
50.17\end{array}$} & \multicolumn{2}{|c|}{ VIG } & \multirow{2}{*}{$\frac{\bar{x}}{50.05}$} & \multirow{2}{*}{$\frac{\sigma}{0.25}$} & \multirow{2}{*}{$\frac{\sigma(\%)}{0.50}$} & \multirow{2}{*}{$\frac{\mathrm{GC}^{\mathrm{a}}}{50.00}$} \\
\hline & 50.00 & 49.90 & & & & & 49.55 & 49.75 & & & & \\
\hline $\mathrm{TiO}_{2}$ & 1.43 & 1.44 & 1.53 & 1.45 & 1.43 & 1.43 & 1.45 & 1.48 & 1.46 & 0.04 & 2.60 & 1.44 \\
\hline $\mathrm{Al}_{2} \mathrm{O}_{3}$ & 15.86 & 16.01 & 15.83 & 15.61 & 15.82 & 15.84 & 16.09 & 15.97 & 15.84 & 0.14 & 0.88 & 15.82 \\
\hline $\mathrm{FeO}^{*}$ & 9.51 & 9.60 & 9.56 & 9.41 & 9.41 & 9.37 & 9.93 & 9.75 & 9.52 & 0.17 & 1.8 & 9.54 \\
\hline $\mathrm{MgO}$ & 8.14 & 8.06 & 7.89 & 8.44 & 7.92 & 8.14 & 8.15 & 8.21 & 8.11 & 0.20 & 2.5 & 8.13 \\
\hline $\mathrm{CaO}$ & 12.05 & 12.12 & 12.03 & 12.01 & 11.90 & 12.08 & 11.76 & 11.70 & 11.97 & 0.14 & 1.1 & 11.96 \\
\hline $\mathrm{Na}_{2} \mathrm{O}$ & 2.49 & 2.41 & 2.47 & 2.67 & 2.60 & 2.42 & 2.71 & 2.75 & 2.56 & 0.13 & 5.0 & 2.59 \\
\hline $\mathrm{K}_{2} \mathrm{O}$ & 0.19 & 0.19 & 0.24 & 0.20 & 0.18 & 0.22 & 0.24 & 0.24 & 0.21 & 0.03 & 12.0 & 0.19 \\
\hline $\mathrm{MnO}$ & 0.17 & 0.17 & 0.17 & $(0.20)$ & 0.16 & 0.17 & 0.17 & 0.17 & 0.17 & $0.00_{4}$ & 2.6 & 0.19 \\
\hline $\mathrm{P}_{2} \mathrm{O}_{5}$ & 0.14 & 0.16 & 0.15 & & 0.11 & 0.14 & & & 0.14 & $0.02^{4}$ & 14.0 & 0.14 \\
\hline $\mathrm{S}^{2}$ & & & & & 0.06 & & & & & & & \\
\hline Total & 99.98 & 100.06 & 99.98 & 100.00 & 99.99 & 99.98 & 100.05 & 100.02 & & & & 100.00 \\
\hline
\end{tabular}

${ }^{\mathrm{a}}$ Shipboard analysis of Sample 396B-7-1, 53-55 cm. 
TABLE 5

Dry-Reduced Analyses of Leg 46 Basalt Standard \#2-Sample 396B-15-2, 120-133 cm

\begin{tabular}{|c|c|c|c|c|c|c|c|c|c|c|c|c|}
\hline \multirow[b]{2}{*}{$\mathrm{SiO}_{2}$} & \multicolumn{2}{|c|}{$\mathrm{COB}$} & \multirow{2}{*}{$\frac{\mathrm{KAN}}{50.29}$} & \multirow{2}{*}{$\frac{\mathrm{UN}-\mathrm{I}}{50.02}$} & \multirow{2}{*}{$\begin{array}{c}\text { JSC } \\
50.38\end{array}$} & \multirow{2}{*}{$\begin{array}{l}\text { R-UB } \\
50.40\end{array}$} & \multicolumn{2}{|c|}{ VIG } & \multirow{2}{*}{$\begin{array}{c}\bar{x} \\
50.16\end{array}$} & \multirow{2}{*}{$\begin{array}{c}\sigma \\
0.23\end{array}$} & \multirow{2}{*}{$\begin{array}{r}\sigma(\%) \\
0.45\end{array}$} & \multirow{2}{*}{$\frac{\mathrm{GC}^{\mathrm{a}}}{50.56}$} \\
\hline & 50.03 & 50.09 & & & & & 49.91 & 49.74 & & & & \\
\hline $\mathrm{TiO}_{2}$ & 1.66 & 1.66 & 1.80 & 1.66 & 1.70 & 1.64 & 1.64 & 1.69 & 1.69 & 0.06 & 3.4 & 1.65 \\
\hline $\mathrm{Al}_{2} \mathrm{O}_{3}$ & 15.50 & 15.50 & 15.55 & 15.36 & 15.39 & 15.48 & 15.69 & 15.73 & 15.50 & 0.12 & 0.81 & 15.18 \\
\hline $\mathrm{FeO}^{*}$ & 10.31 & 10.24 & 10.02 & 10.07 & 9.93 & 10.05 & 10.55 & 10.41 & 10.14 & 0.20 & 2.0 & 10.21 \\
\hline $\mathrm{MgO}$ & 8.22 & 8.30 & 8.11 & 8.59 & 8.18 & 8.13 & 7.98 & 8.22 & 8.23 & 0.19 & 2.3 & 8.04 \\
\hline $\mathrm{CaO}$ & 11.15 & 11.15 & 10.80 & 11.00 & 10.82 & 11.11 & 10.79 & 10.75 & 10.94 & 0.17 & 1.5 & 11.16 \\
\hline $\mathrm{Na}_{2} \mathrm{O}$ & 2.67 & 2.60 & 2.92 & 2.97 & 3.05 & 2.73 & 3.10 & 3.12 & 2.90 & 0.19 & 6.4 & 2.72 \\
\hline $\mathrm{K}_{2} \mathrm{O}$ & 0.13 & 0.13 & 0.18 & 0.13 & 0.12 & 0.14 & 0.18 & 0.18 & 0.15 & 0.03 & 17.0 & 0.14 \\
\hline $\mathrm{MnO}$ & 0.16 & 0.16 & 0.16 & (0.19) & 0.15 & 0.16 & 0.15 & 0.15 & 0.16 & $0.00_{5}$ & 3.4 & 0.17 \\
\hline $\mathrm{P}_{2} \mathrm{O}_{5}$ & 0.14 & 0.15 & 0.18 & & 0.13 & 0.15 & & & 0.15 & 0.02 & 14.0 & 0.17 \\
\hline & & & & & 0.12 & & & & & & & \\
\hline Total & 99.97 & 99.98 & 100.00 & 99.99 & 99.97 & 99.99 & 99.99 & 99.99 & & & & 100.00 \\
\hline
\end{tabular}

a Shipboard analy sis of Sample 396B-15-2, 129-131 cm

TABLE 6

Dry-Reduced Analyses of Leg 46 Basalt Standard \#3 -Sample 396B-22-3, 28-38 cm

\begin{tabular}{|c|c|c|c|c|c|c|c|c|c|c|c|}
\hline & \multicolumn{2}{|c|}{$\mathrm{COB}$} & \multirow{2}{*}{$\frac{\text { KAN }}{49.84}$} & \multirow{2}{*}{$\frac{\text { UN-I }}{49.99}$} & \multirow{2}{*}{$\begin{array}{c}\text { JSC } \\
49.77\end{array}$} & \multirow{2}{*}{$\frac{\text { R-UB }}{49.84}$} & \multicolumn{2}{|c|}{ VIG } & \multirow{2}{*}{$\begin{array}{c}\bar{x} \\
49.72\end{array}$} & \multirow{2}{*}{$\begin{array}{c}\sigma \\
0.23\end{array}$} & \multirow{2}{*}{$\begin{array}{c}\sigma(\%) \\
0.46\end{array}$} \\
\hline $\mathrm{SiO}_{2}$ & 49.31 & 49.49 & & & & & 49.65 & 49.33 & & & \\
\hline $\mathrm{TiO}_{2}$ & 0.99 & 0.98 & $1.70^{1}$ & 0.99 & 0.99 & 0.97 & 1.03 & 1.03 & 1.11 & 0.29 & 26.0 \\
\hline $\mathrm{Al}_{2} \mathrm{O}_{3}$ & 18.37 & 18.31 & 17.86 & 17.66 & 18.10 & 18.07 & 18.21 & 18.28 & 18.05 & 0.25 & 1.4 \\
\hline $\mathrm{FeO}^{*}$ & 7.77 & 7.77 & 7.19 & 7.42 & 7.70 & 7.71 & 8.01 & 8.00 & 7.72 & 0.21 & 2.7 \\
\hline $\mathrm{MgO}$ & 8.27 & 8.14 & 8.06 & 8.63 & 8.00 & 8.16 & 8.22 & 8.35 & 8.23 & 0.25 & 3.0 \\
\hline $\mathrm{CaO}$ & 12.72 & 12.64 & 12.38 & 12.38 & 12.50 & 12.53 & 11.90 & 12.02 & 12.40 & 0.24 & 2.0 \\
\hline $\mathrm{Na}_{2} \mathrm{O}$ & 2.15 & 2.25 & 2.43 & 2.58 & 2.51 & 2.32 & 2.59 & 2.59 & 2.44 & 0.15 & 6.3 \\
\hline $\mathrm{K}_{2} \mathrm{O}$ & 0.18 & 0.18 & 0.23 & 0.19 & 0.18 & 0.17 & 0.23 & 0.24 & 0.20 & 0.03 & 13.3 \\
\hline $\mathrm{MnO}$ & 0.13 & 0.13 & 0.14 & $(0.15)$ & 0.13 & 0.13 & 0.14 & 0.14 & 0.13 & $0.00_{5}$ & 4.2 \\
\hline $\mathrm{P}_{2} \mathrm{O}_{5}$ & 0.09 & 0.09 & 0.14 & & 0.07 & 0.09 & & & 0.10 & $0.03^{\circ}$ & 30.0 \\
\hline $\mathrm{s}^{2}$ & & & & & 0.02 & & & & & & \\
\hline Total & 99.98 & 99.98 & 99.97 & 99.99 & 99.97 & 99.99 & 99.98 & 99.98 & & & \\
\hline
\end{tabular}




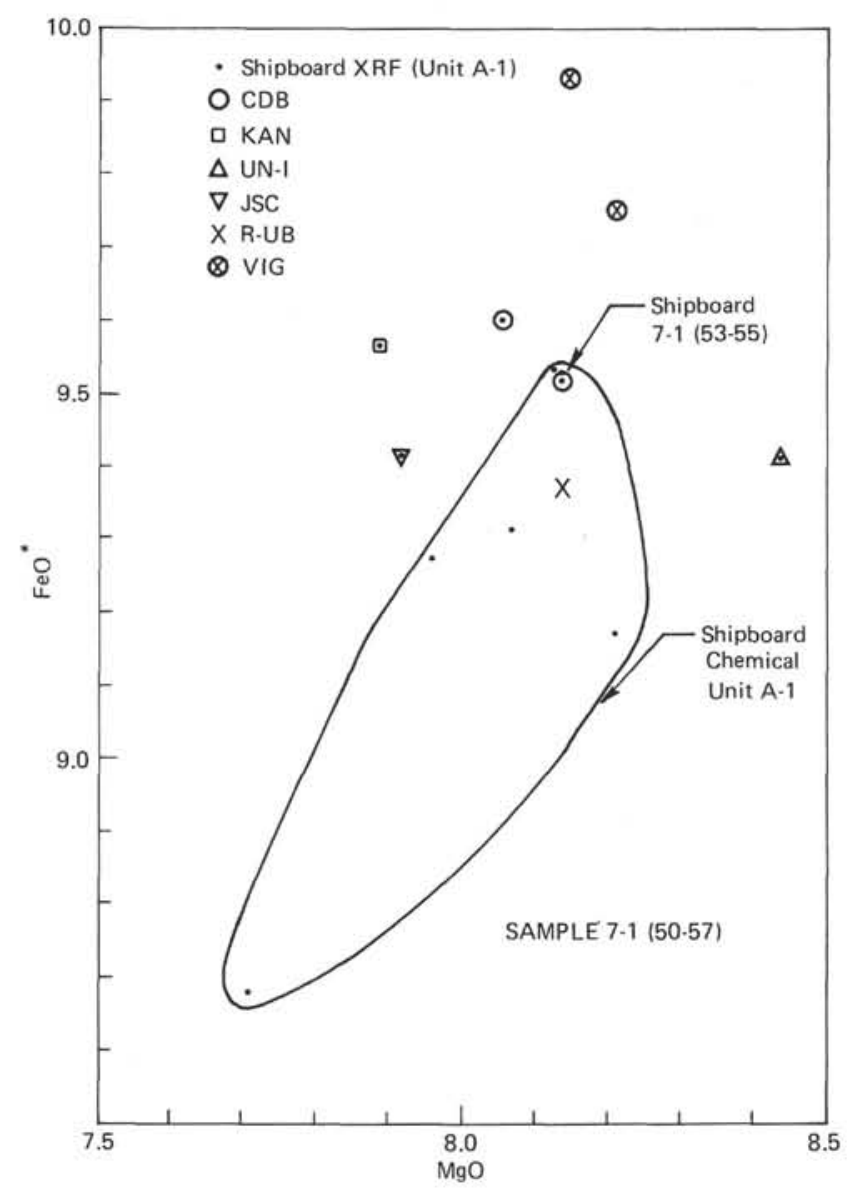

Figure 1. Normalized $\mathrm{FeO}$ * versus $\mathrm{MgO}$ for the interlaboratory standard, $7-1,50-57 \mathrm{~cm}$, along with the shipboard analyses of chemical unit $A-1$ to which that sample belongs.

TABLE 7

Trace Element Concentrations in DSDP Leg 46 Basalt Standards (ppm)

\begin{tabular}{|c|c|c|c|c|c|c|}
\hline & \multicolumn{2}{|c|}{$7-1,50-57 \mathrm{~cm}$} & \multicolumn{2}{|c|}{$15-2,120-133 \mathrm{~cm}$} & \multicolumn{2}{|c|}{$22-3,28-38 \mathrm{~cm}$} \\
\hline & JSC & KAR & JSC & KAR & JSC & KAR \\
\hline $\mathrm{Li}$ & & 11 & & 7 & & 13 \\
\hline $\mathrm{F}$ & & 181 & & 164 & & 150 \\
\hline $\mathrm{Sc}$ & 38.2 & 38.5 & 38.6 & 39.7 & 31.7 & 31.8 \\
\hline V & & 244 & & 193 & & 156 \\
\hline $\mathrm{Cr}$ & 340 & 343 & 300 & 301 & 370 & 396 \\
\hline $\mathrm{Ni}$ & 150 & 158 & 130 & 133 & 110 & 120 \\
\hline $\mathrm{Zn}$ & & 56 & & 66 & & 50 \\
\hline $\mathrm{Sr}$ & & 125 & & 143 & & 110 \\
\hline $\mathrm{La}$ & 2.88 & 2.6 & 3.65 & 4.0 & 1.89 & 2.4 \\
\hline $\mathrm{Ce}$ & 10.0 & 9.5 & 12.5 & 11.6 & 6.4 & 6.7 \\
\hline $\mathrm{Nd}$ & & 7.8 & & 11.9 & & 7.4 \\
\hline $\mathrm{Sm}$ & 3.25 & 3.4 & 3.96 & 4.1 & 2.33 & 2.4 \\
\hline $\mathrm{Eu}$ & 1.18 & 1.15 & 1.41 & 1.39 & 0.89 & 0.83 \\
\hline $\mathrm{Tb}$ & 0.84 & 0.87 & 1.0 & 0.96 & 0.60 & 0.60 \\
\hline Dy & & 5.5 & & 6.7 & & 3.5 \\
\hline $\mathrm{Yb}$ & 3.2 & 2.8 & 3.8 & 3.2 & 2.2 & 2.2 \\
\hline $\mathrm{Lu}$ & 0.49 & 0.50 & 0.55 & 0.57 & 0.36 & 0.38 \\
\hline Hf & 2.4 & 2.5 & 3.1 & 2.9 & 1.6 & 1.5 \\
\hline
\end{tabular}

